\title{
Los estilos de enseñanza de la Educación Física y el Deporte a través de 40 años de vida profesional Teaching styles of Physical Education and Sport through 40 years of professional life Miguel Ángel Delgado Noguera \\ Universidad de Granada (España)
}

Resumen. El objetivo de este artículo es revisar la evolución de los estilos de enseñanza en España, desde la perspectiva de un docente que ha trabajado e investigado el tema durante 40 años de vida profesional. Pretende reflexionar sobre las fases de su vida profesional, al tiempo que analiza su desarrollo personal y como docente ligado a los estilos de enseñanza que estudia, practica, enseña e investiga sobre los mismos. Paralelamente se detiene en las patologías docentes que aparecen en cada fase provocando cambios en su forma de enseñar. Finalmente plantea algunas conclusiones sobre la evolución seguida y las perspectivas de futuro de los estilos de enseñanza.

Palabras claves. Estilos de Enseñanza, vida profesional, investigación.

Abstract. This work is the essay on the evolution of teaching styles in Spain, from the perspective of a teacher of 40 years of professional life. The aim of this article is to review the evolution of teaching styles in Spain, from the perspective of a teacher who has worked and researched the subject for 40 years of professional life. Some thoughts about the stages of his career, while analyzing their personal development and teacher linked to teaching styles studying, practice, teaching and research on them. Parallel stops in teaching conditions that appear in each phase causing changes in their teaching. Finally presents some conclusions about the evolution followed and future prospects of teaching styles.

Keywords. Teaching Styles, professional, research.

\section{Introducción}

Después de cuarenta años de profesión y estando jubilado, en la etapa de la liberación y del distanciamiento emocional de la misma, uno puede reflexionar sobre lo que ha sido su vida profesional a lo largo de esta maratón como docente. Una de esas reflexiones, desde la tranquilidad y la perspectiva de los años, es que mi vida profesional como docente ha estado ligada a la evolución de los Estilos de Enseñanza(EE) en la Educación Física(EF). No creo que haya sido un excelente docente pero sí aprecio que, salvo un periodo de gestión universitaria, sólo he impartido clases, lo mejor que he sabido, al servicio de mis estudiantes.

En estos días se está poniendo de moda, entre los cantantes, después de tantos años de ejercicio profesional, una ansiedad o especie de miedo escénico ante el público que les hace, en algunos casos, abandonar su actividad. Esta experiencia la estoy viviendo ahora, de tal forma que cuando ya no es habitual impartir clases me encuentro incómodo ante la clase. ¿A qué puede ser debido este hecho? Creo que la responsabilidad ahora es mayor. Ya no puedes decir: «aun tengo tiempo para aprender para otras clases», ya que tu actividad docente cotidiana ha finalizado. Esto mismo me está ocurriendo cuando escribo estas líneas, y espero no abandonar.

\section{Analogía del matrimonio y la carrera profesional}

Una de las analogías posibles de mi carrera profesional puede estar ligada a la vida matrimonial y los cambios que se producen en la relación sentimental con tu pareja. Siguen procesos similares. Siempre he comentado que la profesión, como el matrimonio, tiene las siguientes etapas:

1.- La etapa de la poesía, que se manifiesta en los primeros años. Todo es maravilloso. Nuestras ilusiones están intactas y nada es ni se convierte en rutinario. No hay nada normal, todo es extraordinario o así lo vivimos. Todas las experiencias son nuevas. En esta etapa siempre puede haber matadores de ilusiones que digan aquello de: «Sí, así empecé yo», como anticipando que no te hagas muchas ilusiones que tu dedicación a los estudiantes ya descenderá y que el propio sistema ya te irá adocenando y te socializará dentro del mismo. Lo que se conoce como enculturación docente (Delgado Noguera, 2001).

En cuanto a los estilos de enseñanza, esta etapa se caracteriza porque te gusta probar maneras diferentes de impartir la clase. Incluso los estilos más tradicionales se dulcifican porque tú les aportas unas ganas y una ilusión que hasta cuesta reconocer que son unos estilos poco innovadores. A veces vemos docentes que imparten sus clases con

Fecha recepción: 18-12-14- Fecha envío revisores: 18-12-14- Fecha de aceptación: 20-03-15 Miguel Ángel Delgado Noguera

madelgad@ugr.es una asignación de tareas (Estilo de Enseñanza Tradicional) pero su personalidad, su manera de ser, cambia por completo lo esencial de este estilo, la enseñanza es mera instrucción aunque otros factores sociales, afectivos, etc., hacen que sus estudiantes aprendan y además se encuentran satisfechos. De todas formas, en esta etapa experimentas y vas desarrollándote como profesional.

2.- La etapa de la prosa poética. Sigue habiendo momentos ilusionantes pero, poco a poco, se va apoderando de tu vida la rutina, la experiencia no aprendida (se dice que toda experiencia no reflexionada no sirve para nada) y surge cierta desilusión dentro de la normalidad. La duración de esta etapa puede variar en función de factores externos y también de la propia motivación intrínseca. Los proyectos nuevos, los retos, ayudan mucho a vivir la vida personal y profesional con grandes dosis de ilusión renovada. Sin darnos cuenta nos metemos en la siguiente etapa. Durante esta etapa te vas sintiendo más a gusto con cierto estilo de enseñanza y, salvo algunas ocasiones, no intentas enseñar de una manera más innovadora. Parece que has encontrado tu manera acorde a tu forma de pensar en la enseñanza, tus teorías implícitas de la enseñanza y vas simultaneando tu estilo de forma preferente $y$, de vez en cuando, introduces otros estilos de enseñanza. Se da una mezcla de rutina y de innovación.

3.- La etapa de la prosa. La vida se va convirtiendo en rutina. El quehacer cotidiano no conlleva ninguna o escasamente ocasiones nuevas, innovadoras. Esta etapa puede durar demasiado tiempo en la profesión, tanto como en el matrimonio. Ya sólo pasan los años y la ilusión se va perdiendo paulatinamente. Puede aparecer algún momento especial, pero en seguida viene el quehacer rutinario y la obligación de cumplir; le falta poesía e ilusión en tu vida. Es terrible pensar que ya te lo dijeron pero no hemos puesto los medios para que la rutina no dominara nuestra actividad profesional. Inevitablemente se va produciendo un distanciamiento que lleva a una etapa crítica, en la que incluso te cuestionas dónde estoy, cuál es mi papel como docente.

En tu forma de enseñar se va imponiendo un estilo de enseñanza que consideras que se ajusta a tu centro, a tus estudiantes, a tu manera de enseñar y tus concepciones sobre la enseñanza de la EF. En consecuencia, tu forma de abordar la clase se convierte en rutinaria. Siempre aplicas el mismo estilo de enseñanza. No te quieres complicar la vida docente y es que el sistema y lo que te rodea hace que cada vez te limites más a cumplir lo justo. Eres un funcionario al que no le vas a pedir nada más que lo estrictamente necesario y la culpa de tu frustración siempre la tienen otros factores externos.

4.- La etapa de la crisis. Llegado a este momento, se producen dos reacciones: la separación, el abandono o, por el contrario, volverte a enamorar. En la vida profesional, rara vez se deja la profesión docente pero sí se produce abandono de tus obligaciones, le dedicas más tiempo a otras cuestiones no relacionadas con la enseñanza y la educación (asuntos administrativos, excedencias para otras actividades). Habrá 
excusas para todo. Pero lo que más interesa es volver a enamorarte de la misma que ya no es la misma. El tiempo nos ha cambiado a todos. Con lo cual hay que volver a ilusionarte con tu vida profesional y llegar a sentir que estás iniciando una nueva etapa de poesía. Es posible. En los estilos de la Educación Física y el Deporte, esta forma de crisis se manifiesta cuando decides intentar nuevas experiencias didácticas.

\section{Fases de la vida profesional docente}

Desde mi trayectoria profesional y atendiendo a mi currículum vital, intento reflexionar sobre los estilos de enseñanza y cómo han evolucionado y, al mismo, tiempo, qué influencia han tenido en mi enseñanza diaria.

Las diversas fases en la carrera docente son estudiadas por Huberman (1989, 1990), Rosales (1992), Fernández Cruz (1995), Delgado Noguera (1999) y Zabalza y Zabalza (2011). Entre ellas, podemos considerar las siguientes: Fase inicial; fase de estabilización; fase de experimentación; fase crítica; fase de serenidad; fase conservadora y fase de liberación. Ver tabla 1. Se recogen la denominación de las fases o etapas de la profesión, el periodo aproximado de años que abarca y la edad que tenía como docente.

\begin{tabular}{|c|c|c|c|c|}
\hline FASE & NOMBRE & PERÍODO & DURACIÓN & EDAD aproximada \\
\hline $1^{\mathrm{a}}$ Fase & Inicial & $1-2$ años & 2 años & $23-25$ años \\
\hline $2^{\mathrm{a}}$ Fase & Estabilización & $3-7$ años & 5 años & $26-30$ años \\
\hline $3^{\mathrm{a}}$ Fase & Experimentación & $8-15$ años & 8 años & $31-38$ años \\
\hline $4^{\mathrm{a}}$ Fase & Crítica & 16 - 23 años & 8 años & $39-46$ años \\
\hline $5^{\mathrm{a}}$ Fase & Serenidad & $24-32$ años & 9 años & $47-55$ años \\
\hline $\begin{array}{l}6^{6^{a}} \text { Fase } \\
7^{\mathrm{a}} \text { Fase }\end{array}$ & $\begin{array}{l}\text { Conservadora } \\
\text { Liberación }\end{array}$ & $\begin{array}{l}33-37 \text { años } \\
38-42 \text { años }\end{array}$ & $\begin{array}{l}5 \text { años } \\
5 \text { años } \\
42 \text { AÑOS }\end{array}$ & $\begin{array}{l}56-60 \text { años } \\
61-65 \text { años }\end{array}$ \\
\hline
\end{tabular}

Si bien en la Tabla 1 se recogen las fases en base a mi experiencia profesional, evidentemente, en otros casos la duración de las fases y la edad dependerán de múltiples factores. Sólo nos sirven como referencia. Alguna fase puede reducirse en su duración y, en ocasiones, otra fase tiene una duración más prolongada.

Fase inicial o la ilusión por la enseñanza. (2 años). 23-25 años de edad. 1972-1974

La fase inicial o del comienzo en el trabajo profesional responsable constituye una etapa difícil en la que se dan diversos matices. Por un lado, es un choque con la realidad de la enseñanza, se contrasta la teoría con la práctica y se comprueba que muchas de las materias estudiadas en la carrera no son trasladables a la práctica. Por otro lado, se tiene la satisfacción de ser el profesor de unos alumnos y alumnas con los que aplicas tu programación y dispones de autonomía profesional. Se va adquiriendo un conocimiento práctico docente y de la profesión sobre una realidad concreta profesional (Delgado Noguera, 1999). Lo más destacable de esta fase es el choque que se produce entre los conceptos ideales que el profesorado lleva (sus expectativas), con la propia realidad escolar y docente. Como todo proyecto nuevo al que las personas nos enfrentamos cotidianamente, lo normal es afrontarlo con miedo, incertidumbre y sensación de incapacidad que muchas veces nos impide dar cuenta de las nuevas posibilidades que están por venir en nuestra carrera profesional.

En esta fase inicial, durante el periodo 1972-1974, en mi vida profesional tuve una formación inicial escasa, casi nula, en bibliografía sobre la enseñanza y los estilos de enseñanza de la Educación Física. Tuve la suerte de tener unos magníficos profesores que dominaban la enseñanza directiva, instructiva y también algunos ejemplos de profesores que utilizaban una metodología menos directiva. Los primeros conocimientos sobre los estilos de enseñanza los obtuve con la asignatura de metodología I y II, que impartía el profesor Sánchez Bañuelos, con el que trabajé como profesor auxiliar de Metodología de la Enseñanza. Nos presentaba los estilos de enseñanza de Mosston (1966), que traducía de su libro original. En España era la primera vez que en la formación inicial del profesorado de EF se nos presentaba el «spectrum» o espectro de los estilos de enseñanza de Mosston, basados en un proceso de eman- cipación del alumno desde unos estilos de enseñanza donde todas las decisiones eran adoptadas por el profesor hacia una progresión donde se permitía una mayor implicación del alumno en lo que se realizaba. Lo normal es que el profesor diera una orden y los estudiantes sólo tenían que responder a esta orden. Jamás te decía el profesor para qué realizabas tal o cual ejercicio o tarea motriz. Se entendía que el profesor te manda algo que sería bueno para ti y te correspondía obedecer.

Era la primera vez que conocías que, además de realizar los ejercicios según un modelo, podría hacerlos buscando la forma de ejecutar los mismos sin que previamente te dejaran completamente claras las instrucciones o demostraciones para que, como alumno o aprendiz, sólo las reprodujeras. Esto era la teoría, pero en la práctica sucedía de otra manera. Los alumnos estaban acostumbrados a recibir órdenes, pues la época en que estábamos en España era el reflejo exacto ideal para aplicar estilos de enseñanza basados en la orden y el acatar la misma. Los alumnos te demandaban que les dijeras lo que tenían que hacer en la práctica sin más.

Además de mi labor como profesor auxiliar en el INEF de Madrid, tuve la oportunidad de iniciar mi vida profesional como profesor de EF en el colegio de la institución San Isidoro, colegio de huérfanos de periodistas. Fui profesor de gimnasia, según el director del centro y, en esos años, para la mayoría de los alumnos y padres. En este centro pude poner en práctica los estilos de enseñanza que en la teoría había aprendido y simplemente decirles a los alumnos que cada uno realizara la carrera del calentamiento a su ritmo, en lugar de al ritmo que marcaba el profesor, y eso ya era un avance extraordinario. De hecho cuando les indicabas que cada uno hiciera los ejercicios que quisieran se quedaban mirándose los unos a los otros sin saber quéhacer. No estaban acostumbrados a que se les dijera que hicieran algo libremente. Me recuerda el comic de Tonucci (1974), cuando en una de sus viñetas, muestra a un niño al que le dicen: ¡hazlo así!, ¡se hace así!, jsiempre debes hacerlo así!, y luego le dicen, Elige tú; y el niño piensa ¿Elige, elegir? y ¿Qué quiere decirelegir?

Tenía la suerte de dar clases a chicos desde los seis años, ciclo inicial de la Educación General Básica (EGB), pasando por el Bachillerato Unificado Polivante (BUP), hasta los diecisiete años, con el Curso de Orientación Universitaria (COU) y, desde el primer momento, me propuse que los estilos de enseñanza los iría aplicando en función del grado de madurez del grupo. Esta era mi realidad; en los otros centros a los que asistía como profesor para las prácticas didácticas podía comprobar que el estilo de enseñanza casi único era el mando directo y, en ocasiones, un mando directo suavizado acorde a la personalidad del profesor.

La Ley de Educación vigente en estos años es la Ley General de Educación (1970), una ley tardofranquista pero con grandes avances, en algunos aspectos educativos. Habría que aclarar que en la sociedad, a las clases de EF mayoritariamente las denominaban clases de Gimnasia, como antes comenté. La mayoría de los profesores empezaron a clarificar que sus clases no eran de Gimnasia sino de Educación Física. Fue difícil cambiar la denominación durante las dos primeras etapas de mi vida profesional.

En este periodo de mi vida profesional no publiquénada. Era difícil publicar. Hoy día, en los dos primeros años de experiencia docente, encuentras docentes, especialmente universitarios, que han publicado decenas de artículos en revistas nacionales e internacionales y algún libro sobre la enseñanza de la EF. Podemos encontrar autores de publicaciones que escriben sobre la enseñanza de la EF en la escuela sin haber dado ninguna clase en la misma. ¿Es esto lo normal en la actualidad o lo normal sería que se publicara cuando hubieran pasado unos años y se pudiera contrastar lo que se escribe con el desarrollo profesional?

Entiendo que esta fase facilita el hecho de que los profesores principiantes tengan ilusión por la enseñanza y esas ganas de vivir la enseñanza de la EF y que puedan creer que ellos son los que van a cambiar la realidad educativa sin más que su labor docente.

Como patología típica de esta etapa podemos considerar el sentirse «iluminados» dentro de la EF. Esta idea mesiánica es un error. Pensar que sólo con nuestra actuación en las clases vamos a cambiar la educación. Esto es una tarea conjunta de todos los docentes del centro y de las 
familias. Recordar el proverbio africano que dice: «Senecesita a toda una tribu para educar a un niño»

A veces, también se dan otras «enfermedades» como «la utopía de la actitud hacia el deporte». Pensar que con sólo dos horas lectivas de clase de EF vamos a cambiar la actitud de nuestros alumnos y además pensar que «per se» el deporte va a transformar la realidad educativa.

Aunque asociamos ciertas patologías a etapas evolutivas de la carrera docente, éstas se pueden dar en otras etapas diferentes de la vida docente. A veces, los iluminados se aparecen cuando, de pronto, se convierten en nuevos conversos de algunas de las tendencias educativas.

Fase de estabilización docente (5 años). 26-30 años de edad. 1974-1979

En ella se coge confianza en tus propias posibilidades, se afianza tu status administrativo y se establecen unas relaciones más consolidadas con los compañeros de profesión y con el alumnado. Se adquiere un mayor dominio didáctico y conocimiento práctico docente. Se entra en una fase de cierto equilibrio profesional. Eres funcionario de carrera con destino definitivo y ello te hace centrarte definitivamente en tu profesión.

La fase de estabilización abarca en mi vida profesional desde 1974 hasta 1979. Esta etapa acaba cuando tengo siete años de docente en el INEF de Madrid y en mi colegio San Isidoro. En estos cinco años me consideran como profesor de $\mathrm{EF}$ y ya no tienes que pelear para que te compren material para las clases y tus compañeros docentes te reconocen como profesional aunque no tenga la plaza como licenciado. El prestigio lo ganas con tu trabajo diario en las clases, la opinión de los alumnos y el reconocimiento de las familias.

En este tiempo tus clases no están centradas sólo en los estilos tradicionales (mando directo y asignación de tareas), empiezas a trabajar con enseñanza recíproca, los grupos reducidos para el momento de la evaluación inicial y final del curso. Te atreves a dar algunas clases por descubrimiento guiado y resolución de problemas (las menos). Las clases, según estos estilos de enseñanza, varían según los cursos, así con los niños de seis y siete años planteas clases de una especie de modificación de la resolución de problemas motrices muy sencillos, basados en el libro de Liselott Diem (1969) ¿quién es capaz de...? También con estilos de enseñanza más tradicionales como la asignación de tareas, pero planteados como reto motriz. Durante todo el ciclo inicial estos eran los estilos de enseñanza: asignación de tareas o juegos y tareas sencillas a descubrir o buscar variantes del ejercicio.

En el ciclo medio, de ocho a diez años, se trataba de que aprendieran determinados patrones motrices relacionados con los desplazamientos, saltos, giros, etc. El estilo predominante era reproductivo: asignación de tareas con algunos intentos de individualizar con el trabajo por grupos. Es la etapa donde, la mayoría de los autores consideraban que estaban los periodos críticos de aprendizaje y la enseñanza estaba basada fundamentalmente en una instrucción directa o enseñanza instructiva.

En el ciclo superior, con 11, 12 y 13 años, y con la lectura del libro de Mosston (1978) «La enseñanza de la EF. Del comando a descubrimiento», intentaba realizar algunos estilos, tratando de individualizar la enseñanza mediante unos grupos por niveles o por intereses; en otros momentos hacías tus intentos de enseñanza recíproca, como estilo participativo, y era más problemático aplicar estilos de enseñanza productivos, como el descubrimiento guiado y la resolución de problemas.

Ni siquiera en los tres años del Bachillerato Unificado Polivalente (BUP) me atrevía a aplicar de forma generalizada estilos de enseñanza más innovadores. Lo que más conseguí, cuando el horario lo permitía, es que los alumnos de estos cursos me ayudaran en mis clases con los cursos inferiores, les permitía que hicieran de profesores en los deportes en los que estos alumnos destacaban. Erauna experienciamuy gratificante. En el (COU) curso de orientación universitaria, la clase era optativa y me permitía evaluar mi labor de varios años en función de los alumnos que asistían a clase de forma voluntaria. Con estos alumnos de gran madurez académica y personal les planteaba una enseñanza individualizada con algunos programas individuales de condición física y, en ocasiones, los contenidos de las clases a impartir eran decididos por ellos mismos. Realmente podía llevar a cabo con estos cursos, lo que Mosston (1978) denominaba proceso de emancipación. Los estilos de enseñanza del profesorado más utilizados eran fundamentalmente los clásicos, los tradicionales o reproductivos. Para aplicar o avanzar en los estilos de enseñanza había que creer en los alumnos.

En esta etapa, después de siete años de profesión, sólo publiqué un artículo en colaboración de otros profesores, (Sánchez, Delgado Noguera y otros, 1976). Entre ellos, mi maestro Fernando Sánchez Bañuelos, con el que trabajaba en la asignatura de Metodología de la Enseñanza, en el INEF de Madrid. Hoy cualquier profesor novel en la universidad ha escrito más publicaciones que en mis primeros diez años de profesión.

Por eso, en esta etapa se pueden considerar como típicas las patologías de la «inmadurez individual y social permanente», ya que el profesor no conoce o no cree en sus alumnos. Se encuentra con situaciones didácticas que les hacen masificar la enseñanza y desarrollan la misma actividad para todos y con un nivel de ejecución medio (del alumno prototipo) sin considerar las diferencias individuales. También por las creencias o teorías implícitas del profesorado, no cabía la idea de que cada alumno trabajara a su ritmo. Por eso era frecuente en esta época que vieras pocos avances en los estilos de enseñanza y, además, sólo los profesores que recibían clases de metodología de enseñanza conocían que había otras formas de enseñar. Los profesores eran «reproductores del sistema». Esto era lógico en estos años en la enseñanza en España, con la reminiscencia de los «tics» de la dictadura; aunque en los años setenta se producen grandes cambios en la sociedad española, con la llegada de la democracia. En esta época, apenas se impartían cursos de formación o actualización del profesorado, existían escasas revistas especializadas y se publicaban pocos libros sobre didáctica de la EF.

Fase de experimentación y diversificación (8 años). 31-38 años de edad. 1980-1988

En esta fase de experimentación o diversificación, tras varios años como docente, intentas cambiar tu programa, tratas de perfeccionarte en tu tarea docente con lo que pruebas enseñar contenidos diferentes e incluir otras actividades de aprendizaje, innovar en tu enseñanza, plantear unos estilos de enseñanza más innovadores. Tu conocimiento práctico docente te hace buscar otras soluciones a tu enseñanza. En esta fase se inicia una mayor responsabilidad institucional y administrativa y algunos se promocionan como jefes de estudios, directores, etc.

La fase de experimentación o diversificación abarca en mi vida profesional desde 1980 hasta1988. Esta etapa culmina con quince años de profesión. Hay autores que consideran al profesor como un experto a partir de los diez años de ejercicio profesional, pero siempre que no haya seguido una vida profesional en la que cada curso académico sea una repetición del otro. Puedes llevar quince años de profesión pero realmente puedes tener dos años de experiencia.

En estos años ya se publican muchos libros sobre didáctica de la EF. Destacaría los libros de Sánchez Bañuelos (1984), Piéron (1986) y Siedentop (1986). Muchas editoriales se incorporan a publicar temas de EF. La Didáctica de la EF empieza a perder su carácter especulativo y teórico hacia un conocimiento más profesional y de experiencias docentes. En este período la enseñanza de la educación física estaba basada en una didáctica de producto, centrada en los objetivos y el resultado.

El método seguido en la elaboración de programas renovados de la EGB en EF fue la programación por objetivos para favorecer el desarrollo de los elementos básicos de los factores del movimiento, perceptivos, motrices y psicomotrices. En la estructura del proyecto se distribuyen los contenidos en bloques temáticos: aptitudes perceptivas, aptitudes coordenadas (sic), cualidades físicas, actividades en la naturaleza. Cada uno de ellos subdivididos en temas de trabajo distintos para cada curso. También se proponen actividades opcionales para los tres cursos del Ciclo Superior.

Los centros de formación del profesorado de EF (INEFs) se amplían con la creación de los INEF de Granada y Lérida. Desde 1983 
empiezan a surgir muchos movimientos de reforma de la enseñanza y esto, sin lugar a duda, va cambiar también la enseñanza de la EF.

En lo que respecta a los estilos de enseñanza, en los grupos de reforma y experimentación en la enseñanza se prueban otros estilos de enseñanza alternativos. Pese a que la EF mantenía una enseñanza prácticamente deportiva y gimnastica aparecen nuevos contenidos en la materia. Y estos nuevos contenidos llaman a nuevas formas de enseñar.

En este período se desarrollan dos leyes: La LOECE, 1980 (UCD). Una ley marcada por la constitución y el golpe de estado. Esta Ley, la primera plenamente en democracia, fue elaborada por el gobierno de Adolfo Suárez con la Constitución de 1978 recién estrenada. El PSOE la recurrió por no respetar el espíritu de la Constitución y el Tribunal Constitucional (TC) le dio la razón en bastantes puntos. Tras la sentencia, la UCD debía haber revisado la Ley de forma profunda, pero el golpe de Estado del 23-F y la posterior victoria electoral del PSOE lo evitó. Por ello jamás entró en vigor. La LODE, 1985(PSOE) introduce el concierto educativo.

En 1980 se publica la Ley de cultura física y deportes, donde se impulsa la investigación en ciencias de la EF y el deporte. Un embrión que esperábamos produjera avances en la práctica profesional. Es una etapa muy fructífera en la enseñanza de la EF, pero la realidad es que, en la mayoría de los centros escolares, la enseñanza de la EF era todavía muy tradicional.

Para los profesores jóvenes les era muy fácil su incorporación a las nuevas corrientes curriculares de la EF y la sociedad incitaba a todos a cambiar nuestra forma de enseñar. De hecho, son muchos los cursos de actualización del profesorado que, en estos años, se impartían sobre las nuevas formas de enseñanza, sobre la utilización de estilos innovadores. Estos cambios, en algunos casos, produjo un movimiento pendular que pasaba de una didáctica de producto, basada en los objetivos operativos, hacia una didáctica de proceso, basada en la experiencia «per se», sin que lo importante fuera el resultado sino el proceso seguido durante la clase de EF. Esto lleva a que algunos docentes incorporaban cualquier propuesta de actividad física que no fuera la deportiva sin reflexión, e incluso con la inclusión de deportes autóctonos o nuevos deportes o actividades físicas que, en algunas ocasiones, enseñaban con métodos tradicionales. Era puro esnobismo didáctico. En esta fase se puede hablar de dos patologías: los «Consumidores de modas», que incorporaban cualquier actividad mejor cuanto más alejada de lo que se venía haciendo. También se plantea una mal entendida «Intelectualización de la EF», donde los profesores de EF «copiamos los defectos» más clásicos de los funcionarios docentes de otras áreas (enseñanza más tradicional, una enseñanza pasiva en las aulas, exámenes clásicos, apuntes, etc.) ¡Cuántas formas hay de enseñar los conceptos de nuestra materia que no pasan necesariamente por una enseñanza meramente instructiva y escasamente significativa!

Esta diversificación de la enseñanza nos llevó a que los estilos de enseñanza que se proponían en las clases de EF fueran más variados y podían coexistir la enseñanza reproductiva con estilos individualizadores, participativos y socializadores.

En mis clases pude poner en marcha la enseñanza recíproca o por grupos reducidos, en los que los estudiantes hacían de profesores de sus compañeros y, en ocasiones, se podía individualizar según el nivel de logro, por ejemplo en la enseñanza del voleibol. También podíamos utilizar estilos de enseñanza creativos, en los que se promovía la iniciativa en las respuestas motrices, bien con una solución o con varias alternativas de solución. Estas experiencias las realizaba en el colegio, con alumnos mayores de bachillerato y con los estudiantes del INEF de Madrid y, posteriormente, con los de Granada. Eran pequeños avances en la enseñanza con nuevas experiencias didácticas.

Para mí tuvo una influencia especial el libro relacionado con los estilos de enseñanza, titulado: «Modelos de Enseñanza» de Joyce y Weil (1985). Era una influencia ajena a la ejercida por Mosston desde la $\mathrm{EF}$, y que proporcionaba un aire fresco en cuanto a nuevas propuestas de enseñanza.

Durante este periodo pude publicar artículos sobre la enseñanza individualizada y sobre la microenseñanza, como un estilo participativo, en la revista El entrenador del fútbol, así como en la revista Apunts sobre la evolución de las líneas pedagógicas de la EF en España.

Fase crítica o de cuestionamiento de mi enseñanza (8 años). 39-46 años de edad. 1989-1996

En algún momento de la vida profesional te planteas tu labor educativa en la enseñanza de la EF y esto puede producirse antes o después de los quince años de experiencia docente. Es un cuestionamiento crítico de la propia profesión. A la necesidad de cambiar puede seguir un cuestionamiento crítico de la rutina durante la enseñanza. Esto puede provocar desde abandono temporal o definitivo de la docencia hasta entrar en un período crítico, en muchos casos es negativo y en otros positivo, de relanzar la profesión y cambiar la rutina diaria de la enseñanza. Si la fase anterior es fructífera es probable que no se pase por esta fase.

En mi caso se producen muchos cambios. Uno, que considero negativo, es mi distanciamiento de la realidad de los centros escolares. Ya no imparto clases de EF en un centro escolar, si bien es cierto que tengo contactos esporádicos con los profesores de EF de los centros escolares con las prácticas de enseñanza y durante los cursos de perfeccionamiento del profesorado de EF. Siempre he querido mantener ese contacto, pues de lo contrario podía caer en el desconocimiento de la realidad escolar, que es cambiante. Es una etapa crítica por la que todo docente ha pasado. En mi caso me lleva a publicar «los Estilos de la Enseñanza. Una propuesta para la reforma de la enseñanza» (1991)

Soy muy crítico a la dinámica que la enseñanza de la EF está tomando, pese a que cada vez hay más publicaciones de índole didáctica, tanto de didáctica general como de didáctica específica.

Había muchos movimientos alternativos en la enseñanza y yo quise aportar mi pequeño grano de arena a la construcción de una enseñanza de la EF más sólida, donde los profesores traten de cambiar su forma de enseñar. Teníamos una oportunidad única con la integración de los estudios de EF en la Universidad. Desaparece el INEF de Granada y nace, con mucha ilusión, la primera Facultad. No era fácil y suponía una gran responsabilidad.

Paralelamente se cambia la Ley de Educación (LGE) de 1970. La Ley de Ordenación General del Sistema Educativo (LOGSE, 1990) introduce la escolaridad obligatoria hasta los 16 años, entre otras medidas. Es la Ley que permite a las comunidades autónomas redactar una parte muy importante de los contenidos educativos. Se adapta a un modelo más tecnológico en la educación, introduciéndose la Enseñanza Secundaria Obligatoria (ESO). Creo que fue una oportunidad perdida por la falta de medios, para luego convertirse en una losa sobre el profesorado que más ilusión puso en esta ley.

Esta fase crítica fue fructífera. Para mí el contacto con los estilos de enseñanza se realiza a través de las experiencias prácticas de los estudiantes de Granada y con las primeras investigaciones que dirijo. La primera línea de investigación era la formación inicial del profesorado de EF. En este período se lee mi tesis doctoral sobre la «Influencia de un entrenamiento docente durante las prácticas docentes sobre algunas de las competencias del profesor de Educación Física». Posteriormente vendrían varias tesis sobre esta temática. La segunda línea de investigación fue la didáctica de la EF. En esta etapa destacamos dos tesis relacionadas con los estilos de enseñanza: la investigación de Vernetta (1994), que trabaja un estilo de enseñanza mixto mediante la asignación de tareas con elementos de individualización, empleando tres estrategias en la práctica y la investigación de Generelo (1995), que nos lleva al uso de estilos de enseñanza individualizadores.

Apenas hay investigaciones sobre los estilos de enseñanza en España. Era necesario pasar de una didáctica de la EF especulativa, con buenas intenciones, a una didáctica más científica, tanto desde una perspectiva conductista (racionalista) como de una investigación cualitativa y naturalista. De una didáctica basada en el producto a una didáctica basada en el proceso. Como consecuencia hace que se produzca una desorientación general y, en un movimiento pendular, se pase a una didáctica donde lo importante es pasárselo bien aunque no haya resul- 
tados y no se produzcan aprendizajes.

Nos encontramos en esta fase a «los fundamentalistas», que creyendo que la solución está sólo en una didáctica procesal no se preocupan del aprendizaje y promueven el «aprender a no aprender». Lo importante es que el alumno disfrute de la clase y en la clase aunque no aprendan. Con esta patología se pueden dar cuatro posibilidades: a) aprender sin pasárselo bien - no deseable. b) pasarlo bien sin aprender no deseable. c) No pasarlo bien y no aprender - no deseable. Y, por último, d) aprender y pasarlo bien - esto sería nuestra pretensión y aspiración.

La solución está en una vacuna trifásica que consta de investigación, innovación y trabajo en equipo, que nos llevará a un adecuado desarrollo profesional. Remedio que, en la realidad de nuestras aulas en los centros escolares encuentra muchas dificultades: muchas horas de clases, sin apenas reflexión, cambio continuo de los docentes de centro, escasa potenciación de la investigación e innovación, la falta de promoción profesional y consideración de los méritos adquiridos. De esta fase se sale con una propuesta decidida y un compromiso del personal docente que, si es acompañado con otros compañeros de profesión, supondrá una etapa fructífera (ver figura 1). Han de estar interrelacionada la investigación, la innovación y el trabajo en equipo.

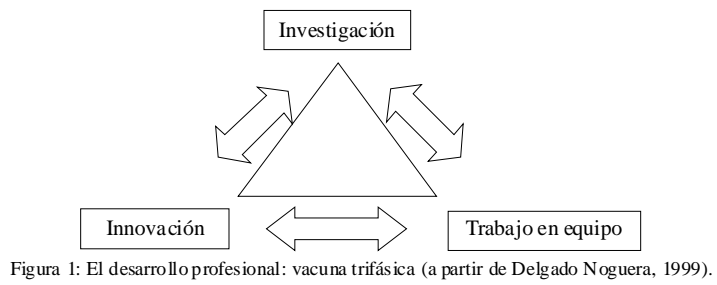

Fase de serenidad y distanciamiento emocional de la enseñanza (9 años). 47-55 años de edad. 1997-2005

Tras muchos años de profesión (alrededor de 30 años) y superada la etapa crítica, el profesorado está más distendido, menos dinámico, con menor implicación afectiva respecto de la enseñanza y de su alumnado y además se inicia una cesión de la responsabilidad administrativa. Este periodo de mi vida profesional abarca desde 1997 hasta 2005. No siempre ocurre que esta etapa sea serena sino que, en algunos casos, es una etapa muy productiva.

La experiencia reflexionada de tantos años te permite dos reacciones: una, positiva, que supone un relanzamiento de la ilusión por la enseñanza y otra, negativa, que nos hace limitarnos a ir pasando años sin dejar huella de nuestra docencia. Es el momento de adecuar los estilos de enseñanza a tu realidad docente.

Entre las patologías de los profesores de esta etapa es frecuente el «freno a la innovación». El problema «ya estoy fijo en mi plaza» es uno de los motivos por los que podemos caer en la rutina. Algunos docentes llegan a la conclusión «haga lo que haga siempre me van a pagar lo mismo» y «para qué me voy a complicar la vida». En algunos casos se dan evidencias del «burn out» o estar quemado de la profesión tanto en esta fase como en la siguiente.

En este periodo se ha avanzado mucho en los estilos de enseñanza. Destacaría cuatro investigaciones muy importantes para el conocimiento de los mismos en los que pude colaborar y a aprender de los investigadores:

Sicilia (1997) estudia la aplicación de tres estilos de enseñanza, desde un estilo reproductivo (tradicional, asignación de tareas y enseñanza recíproca) hasta la microenseñanza. Este estudio permitió la publicación del libro Sicilia y Delgado Noguera (2002).

Méndez (1999) contrasta las dos técnicas de enseñanza fundamentales (instrucción directa e indagación), base para los estilos reproductivos o productivos, y una técnica de enseñanza combinada de las dos anteriores en la enseñanza de dos deportes.

Cuellar (1999) tiene como objetivo principal fue averiguar cómo enseñar mejor una coreografía de danza flamenca mediante la aplicación de dos formas de trabajo: una innovadora, que resulta de la adaptación y conjugación de varios Estilos de Enseñanza (EE) a las características específicas de este tipo de danza (EE Innovador Adaptado) y la que tradicionalmente se utiliza en danza (Mando Directo).

Solana (2003) analiza si los alumnos quieren ser partícipes de su aprendizaje o, por el contrario, ser sujetos pasivos. Es decir, si aceptarán o asumirán ciertas dosis de responsabilidad. Se desarrollaron diferentes estudios participativos y socializadores aplicables a la enseñanza de la Educación Física. Son estudios realizados en el ámbito universitario, aunque en contexto reales de enseñanza en centros escolares. Por ello considero que la investigación es el motor del avance en la enseñanza de la Educación Física (Delgado Noguera, 2005).

Pero la realidad de las aulas, según el estudio de Manzano et al. (2003), es que el profesorado deAndalucía no ha llegado a generalizar la utilización de estilos de enseñanza innovadores, predominando los estilos tradicionales a la hora de dar sus clases de Educación Física. El profesorado se encuentra sereno y seguro con la utilización de los estilos de enseñanza tradicionales y no se plantea modificar su manera de enseñar. No quieren arriesgarse, aunque declaren que los estilos que más le gustan son los estilos innovadores y productivos frente a los estilos tradicionales o reproductivos. Pero la realidad de las aulas muestran una enseñanza más rutinaria, lo que no implica que, en ocasiones, empleen esporádicamente algunas actuaciones más propias de los estilos cognitivos y creativos en algunas de sus clases.

Los estilos de enseñanza son importantes, pero el eje de atención no tiene que estar centrado exclusivamente en el profesorado, siguiendo las líneas de investigación didáctica de los estilos de enseñanza: presagio-producto; proceso-producto y mediación del profesor. Por eso surge el estudio de los estilos de enseñanza desde la perspectiva de los alumnos. Necesitamos conocer cómo median los alumnos en el proceso de enseñanza-aprendizaje y para ello es esencial estudiar los estilos de aprendizaje. Una primera aproximación fue el estudio de Medina y Delgado (1998), donde se estudian los modelos de aprendizaje de los alumnos y sus teorías implícitas acerca de la EF. Posteriormente se establecen relaciones entre los estilos de enseñanza y los estilos de aprendizaje de los alumnos (Sicilia y Delgado Noguera, 2002).

También era necesario acercarse a las aulas y ver qué experiencias, innovaciones y propuestas didácticas realizaban los profesores en sus clases. Un primer intento fueron los trabajos recopilados por Delgado Noguera (1996) y Delgado Noguera, López, Medina y Viciana (1999), que vinieron a recoger las investigaciones en el aula que realizaba el profesorado EF, presentadas en unas jornadas didácticas.

Fase conservadora y crítica de la enseñanza (5 años) 56-60 años de edad. 2006-2011.

En esta fase conservadora y crítica el profesorado se muestra escéptico ante la innovación, los cambios y las nuevas reformas de la enseñanza. Por otro lado, es muy crítico en relación con los nuevos profesores, con sus alumnos/as y contra la política y administración educativa. Este tipo de profesorado puede ejercer una influencia negativa en los profesores más jóvenes.

Como patologías de esta fase, podemos encontrar a los «matadores de ilusiones», que ante la ilusión de los compañeros jóvenes que empiezan a trabajar sueltan frases tan lapidarias como «Sí. Así empecé yo», «Da igual lo que hagas, la administración no te lo reconocerá» y también a los que ponen «freno a la innovación», porque se encuentran seguros con lo que hacen y consideran que ya no van a aprender nada.

Uno de los problemas de la clasificación de los estilos de enseñanza es que, aunque en la realidad de las aulas se desarrollan clases de estilos de enseñanza que pueden ser considerados como socializadores, ya que fomentan la cooperación, etc., estos no estaban definidos. Dentro de los estilos de enseñanza socializadores considero los siguientes: Grupos de roles, Grupos solidarios, Grupos autogestionados, Grupos cooperativos y Grupos con dinámica de grupo (Delgado Noguera, 2008 y 2009).

En estos años también colaboro con los investigadores y seguimos aprendiendo de los estilos de enseñanza. Destacamos tres investigacio- 
nes que tratan de: (1) resolver problemas de convivencia; Gil (2009) busca resolver problemas concretos de aula. Para la resolución de estos conflictos es importante el uso de los estilos de enseñanza socializadores a través de técnicas grupales y estrategias de mediación; (2) Montávez (2011) investiga los estilos de enseñanza utilizados por el profesorado en el contenido de expresión corporal; y, (3) Guedea (2009) estudia los estilos de enseñanza que utilizan los docentes de EF en educación primaria en el estado mexicano de Chihuahua.

Otros estilos de enseñanza emergen y son necesarios estudiarlos; me refiero a la metodología que se utiliza con las nuevas tecnologías. Urge clarificar si este tipo de estilos de enseñanza pueden ser utilizados al más puro estilo tradicional o bien ser unos estilos de enseñanza innovadores. Es evidente que en la sociedad actual los medios de comunicación de masa, internet y las nuevas tecnologías, han impregnado toda la vida de las personas. A ello no es ajena la EF y los profesores, pero se está cayendo en un uso abusivo de herramientas a utilizar, más que favorecer que el individuo se mueva; se le hace estar más tiempo frente al ordenador o al móvil. La EF no puede estar ajena a estos avances, pero no podemos permitir que su uso provoque más sedentarismo. Hay blogs de alumnos en clases de EF que suponen que se está más tiempo sentado, inactivo, después de las clases que realizando actividad física.

Pero también hay que alertar ante nuevas patologías como los «adictos a las Tics», en los que podemos encontrar profesores que se consideran innovadores por utilizar los medios tecnológicos (Delgado, 2009, 2014). Otras patologías nos llevan a una «intelectualización de la Educación Física» donde los contenidos referidos a los conceptos predominan sobre los contenidos procedimentales, motrices y afectivosociales. Quizás la razón esté fundamentada en que, cercanos a los sesenta años, las horas, en el patio, el gimnasio y las pistas polideportivas, se hacen muy duras.

Fase de liberación o disentimiento de la enseñanza (5 años). 61-65 años de edad. 2012-2015

Esta fase se puede dar en la antesala de retirarse de la profesión. Los intereses se repliegan a la familia o sobre uno mismo. Se dedica el tiempo mínimo necesario a la profesión.

Entre las patologías se destaca el "Que investiguen otros» y te refugias o repliegas en otros intereses.

Como nunca uno deja de ser profesor, en algunos casos se prolonga la actividad profesional trabajando en situaciones informales, con ONGs o grupos en los que puedes seguir colaborando en temas relacionados con la Educación Física. En mi caso, ahora gozo la docencia, como colaborador de mi departamento y de mi facultad, pero con una carga horaria muy reducida. Lógicamente, te vas dando cuenta que te vas quedando atrás. Siempre he comentado la frase de Alicia en el País de las Maravillas: «En este país, para estar en el mismo sitio hay que estar corriendo», cuando en la historia, los habitantes del país de la Reina Roja deben correr lo más rápido que puedan, sólo para permanecer donde están, pues el país se mueve con ellos. Es cierto, ahora tu ritmo es más lento, vives la vida más despacio. Además te das cuenta que hay vida fuera de la universidad y descubres muchas cosas de ti como persona y como docente. Ahora eres más crítico contigo mismo pero, por otro lado, eres un espectador de lo que era tu profesión. Detectas muchas cuestiones profesionales que han cambiado y también eres consciente que hay muchas otras que no te gustan. Supongo que es porque la sociedad ha cambiado mucho, incluyendo a los estudiantes, las familias, los centros docentes, etc. Te sientes un poco descatalogado en tus ideas; antes tenía algunas certezas y ahora tengo más dudas. Esto lo he pensado yo muchas veces cuando decía: «Te puedo dar consejos para ayer, pero menos para hoy y no me atrevo para mañana, porque soy tan inexperto como tú». El mejor consejo siempre lo da la experiencia, pero siempre llega demasiado tarde.

Los estilos de enseñanza hoy, más que nunca, no son algo rígido, sino que son una realidad más poliédrica. Son una adaptación reflexiva y crítica de una manera de enseñar acorde con el alumnado, el contenido y las circunstancias contextuales de la enseñanza de la EF. La calidad ha sido siempre una preocupación del docente que ha querido hacer su labor lo mejor posible. Justo en este momento, previo a mi jubilación, surge con más fuerza que nunca la calidad, los modelos de gestión de la calidad y los indicadores de la calidad.

En la EF, López Jiménez (2012) realiza una adaptación del modelo europeo de la calidad a nuestra materia. Elabora cuatro cuestionarios de la Calidad en Educación Física (CALEF): evaluación, profesorado, familia y alumnado. Los indicadores de calidad del proceso de enseñanza, entre los que se encuentran los estilos de enseñanza, ocupan un peso específico de, aproximadamente, un 40\%. Dentro de los indicadores de calidad se consideran los siguientes: a) liderazgo del departamento de EF; b) profesorado; c) aspectos organizativos de la materia; d) procesos; e) percepción de la labor del profesor; f) resultados de los alumnos; g) familia, sociedad y h) rendimientos académicos. Pese a las críticas del modelo, consideramos que el disponer de un modelo de calidad en EF nos ayudará a mejorar y a reflexionar sobre nuestra enseñanza. La evaluación de la calidad docente y los estilos de enseñanza son necesarios para mejorar (López Jiménez y Delgado Noguera, 2010).

Una última investigación, preocupada por la responsabilidad personal y social, abre un camino nuevo para el estudio de los programas de intervención para la mejora del alumno y el tipo de estilo o estilos de enseñanza más acordes para ello (Caballero, 2012).

Queda mucho camino por andar, pero hasta aquí lo que he podido destacar de los estilos de enseñanza en España a través de mi vida profesional. Seguro que existen otros estudios e investigaciones que no he mencionado en este recorrido pero, en ellas no he sido actor principal o secundario de esta evolución de los estilos de enseñanza a través de mis cuarenta años de profesión docente. Alos interesados, recomiendo esta página: http://www.spectrumofteachingstyles.org/ No existe una página web similar en castellano.

\section{A modo de conclusión}

Expongo a continuación, en la tabla 2, la que comparo la Educación Física en mis inicios profesionales y en la actualidad, en base a un listado

Tabla 2.

\begin{tabular}{|c|c|c|}
\hline Elemento Didáctico & AYER & HOY \\
\hline $\begin{array}{l}\text { Tipo de } \\
\text { Conocimiento }\end{array}$ & $\begin{array}{l}\text { Profesional, experiencial, } \\
\text { especulativo }\end{array}$ & Científico, Profesional \\
\hline $\begin{array}{l}\text { Carácter del } \\
\text { Conocimiento }\end{array}$ & Estable. Fijo & En continuo cambio \\
\hline $\begin{array}{l}\text { Forma de } \\
\text { Conocimiento }\end{array}$ & $\begin{array}{l}\text { Libros y algunas revistas. } \\
\text { Apenas se publicaban }\end{array}$ & $\begin{array}{l}\text { Conocimiento digital. Tesis doctorales, } \\
\text { investigac iones de todo tipo, cuantitativas } \\
\text { y cualitativas. Multitud de Revistas } \\
\text { científicas algunas con escasa } \\
\text { transferencia a la Profesión }\end{array}$ \\
\hline Tipo de Didáctica & Experiencia y pre científica & Científica y variada \\
\hline $\begin{array}{l}\text { Enfoque de la } \\
\text { Didáctica }\end{array}$ & Proceso-producto & $\begin{array}{l}\text { Desde el Proceso producto al modelo } \\
\text { ecológico, constructivista, etc. }\end{array}$ \\
\hline $\begin{array}{l}\text { Finalidad de la } \\
\text { Didáctica }\end{array}$ & $\begin{array}{l}\text { Búsqueda de un método } \\
\text { universal. Estilo de enseñanza } \\
\text { eficaz en cualquier contexto }\end{array}$ & $\begin{array}{l}\text { Multip lic idad de Estilos de Enseña nza, } \\
\text { centrada en el contexto de la enseñanza. } \\
\text { No hay EE pueda servir para cualquier } \\
\text { situación didáctica }\end{array}$ \\
\hline Rol del Docente & $\begin{array}{l}\text { Vocacional. Modelo. } \\
\text { Enseñanza instructiva. Rol } \\
\text { transmisor y activo }\end{array}$ & $\begin{array}{l}\text { Funcionarial. Menor implicación externa, } \\
\text { Enseñanza menos instructiva. Mejor } \\
\text { preparados }\end{array}$ \\
\hline $\begin{array}{l}\text { Nuevas tecnologías } \\
\text { de la E.F. }\end{array}$ & $\begin{array}{l}\text { Escasa o nula. Cronómetros y } \\
\text { calculadoras }\end{array}$ & $\begin{array}{l}\text { Irrupción de todo tipo de tecnologías de la } \\
\text { información, medios audiovisuales en el } \\
\text { aula, pulsómetros, blogs, etc. }\end{array}$ \\
\hline $\begin{array}{l}\text { Evaluación de la } \\
\text { E.F. }\end{array}$ & $\begin{array}{l}\text { Resultados. También } \\
\text { Actitudes y conocimientos. } \\
\text { Test. Exámenes. Producto }\end{array}$ & $\begin{array}{l}\text { Integral. Diversidad de métodos de } \\
\text { evaluación: Trabajos, Diarios, } \\
\text { Seguimiento actitud en las c lases, etc. } \\
\text { Cualitativa > Cuantitativa }\end{array}$ \\
\hline $\begin{array}{l}\text { Investigación en la } \\
\text { E.F. }\end{array}$ & $\begin{array}{l}\text { Experiencias didácticas no } \\
\text { publicadas. Investigación en } \\
\text { elaula }\end{array}$ & Investigaciones de todo tipo \\
\hline $\begin{array}{l}\text { Modelo curricular } \\
\text { de la E.F. }\end{array}$ & Fijo & Abierto \\
\hline $\begin{array}{l}\text { Las relaciones de la } \\
\text { Didáctica de la E.F. }\end{array}$ & El profesor es el centro & $\begin{array}{l}\text { Se deshace la preponderancia de los tres } \\
\text { elementos. Ni el centro es el profesor, ni } \\
\text { el centro es la materia ni sólo es el } \\
\text { alumno. }\end{array}$ \\
\hline $\begin{array}{l}\text { Técnica de } \\
\text { Enseñanza } \\
\end{array}$ & Instrucción directa & Mixta. Indagac ión. \\
\hline $\begin{array}{l}\text { Estilos de } \\
\text { Enseñanza }\end{array}$ & Estilos reproductivos & Estilos mix tos. Estilos productivos \\
\hline $\begin{array}{l}\text { Estilo de Enseñanza } \\
\text { preferente }\end{array}$ & Mand o direc to & Combinación de estilos de enseñanza \\
\hline $\begin{array}{l}\text { Las relaciones de } \\
\text { Género }\end{array}$ & $\begin{array}{l}\text { Clases donde las rela ciones } \\
\text { está separadas por los grupos } \\
\text { de chicos y chicas }\end{array}$ & Se pretende una integración natural \\
\hline
\end{tabular}


de diferentes elementos didácticos.

Después de una vida profesional en la que he sido partícipe, observador y estudioso de los estilos de enseñanza puedo concluir lo siguiente respecto a estos:

-La dificultad de estudiar los estilos de enseñanza, que se manifiesta en:

- No acuerdo en las denominaciones de los diferentes estilos.

- Polisemia existente en su denominación.

- Problemática de estos estudios desde una perspectiva única.

- Desnaturalización de la enseñanza cuando se pretende investigar desde un enfoque exclusivamente experimental.

- Escasa transferencia de los estudios e investigaciones sobre los estilos de enseñanza en la práctica profesional.

-Existen muchas experiencias, muy relevantes, realizadas por el profesorado que no son publicadas.

-Necesidad de jornadas de dos tipos: (a) reuniones científicas de investigadores; (b) jornadas de experiencias profesionales. Sobre este tipo de jornadas tenemos algunas experiencias en las que los profesores de secundaria exponen sus novedades, innovaciones e investigaciones sobre los estilos de enseñanza.

-No hay que estudiar los estilos de enseñanza de forma aislada, sino de una perspectiva ecológica y no olvidar los estilos de aprendizaje en la enseñanza de la EF. Se trata de un tópico escasamente investigado.

-La desvinculación de la formación del profesorado con la sociedad actual.

La poca actualización didáctica, formamos hoy, para mañana, con métodos de ayer. http://www.educdoscero.com/2011/10/una-pisca-de-actitud-20-para-ser.html

-Necesidad de más investigación sobre los EE.

-Explorar nuevos estilos de enseñanza adecuados a la nueva realidad social y de la escuela.

Para finalizar, termino con una frase que sintetiza el verdadero lema que tiene que existir en nuestra trayectoria como profesional de la enseñanza: «Mi derecho a no cambiar termina allí donde comienza el derecho de mis alumnos al mejor profesor que llevo dentro, el cual, por definición, nace cada año» (Fernández Pérez, 1994).

No podemos olvidar que la situación actual es fruto de muchos hombres y mujeres que apostaron por la educación física y dignificaron esta profesión y estos estudios. Los profesionales actuales y futuros deberían conocer la evolución que ha sufrido la EF y sentirse responsables en seguir mejorando colectivamente la profesión. Dixit.

\section{Referencias}

Bain, K. (2007). Lo que hacen los mejores profesores universitarios. Valencia: Universitat de valencia. Servei de publicacions.

Caballero, P.J. (2012). Diseño y evaluación de un programa de responsabilidad personal y social a través de la actividad física en el medio natural en alumnos de formación profesional (Tesis doctoral). Sevilla: Universidad de Sevilla.

Cuellar, M.J. (1999). Estudio de la adaptación de los estilos de enseñanza a sesiones de danza flamenca escolar. Un nuevo planteamiento didáctico. (Tesis doctoral). Granada: Universidad de Granada.

Delgado Noguera, M.A. (1996). Formación permanente e Innovación experiencias profesionales en la enseñanza de la educación física. Pp 1-8. En Delgado, M.A.; Medina, J.; Viciana, J. y Gutiérrez (editores) (1996) Formación permanente e Innovación experiencias profesionales en la enseñanza de la educación física. Granada: Centro de Formación Continua. Universidad de Granada.

Delgado Noguera, M.A. y Medina, J.(1997). El Practicum de enseñanza desde la perspectiva de los tutores. Un estudio exploratorio. Colección ICd. Investigaciones en Ciencias del Deporte. Madrid: MEC. Consejo Superior de Deportes.

Delgado Noguera, M.A. (1998). El practicum en la formación del pro- fesorado de EF. Estrategias formativas. Huelva: Servicio Publicaciones. Universidad de Huelva.

Medina, J. y Delgado, M.A. (1998). Una primera aproximación a la investigación sobre las teorías del alumno acerca de la Educación Física. Actas del II Congreso Internacional sobre la Enseñanza de la Educación Física y el Deporte escolar. Almería. Pp: 446-450.

Delgado Noguera, M.A. (1999). Patologías de la enseñanza de la EF y el Deporte. Perspectivas de la actividad física y el deporte. $\mathrm{N}^{\circ}$ 19/ 20. 1999.

Delgado Noguera, M.A. López, P., Medina, J. y Viciana, J. (Coordinadores). (1999). Investigaciones en la práctica de la enseñanza de la Educación Física. Granada: Reprografía Digital Granada. Universidad de Granada.

Delgado Noguera, M.A. (2001). Enculturación en el practicum de enseñanza. Tránsito de alumno a profesor de Educación Física. En Latiesa, M. y Martos, P. (2001). Deporte y cambio social en el umbral del siglo XXI. Volumen II. Madrid: Esteban Sanz.

Delgado Noguera, M.A. (2005). La investigación, motor del avance en la enseñanza de la Educación Física. En González Suárez, A. (editor). Avances en las ciencias del Deporte. Vitoria: Universidad del País Vasco.

Delgado Noguera, M.A. (2008). La Educación Física y los estilos de enseñanza socializadores. Sistemas de evaluación. I Congreso de Educación Física. Ciudad de Ceuta. Nuevas perspectivas de la educación física en el siglo XX. Adaptarse a las innovaciones científicas y al desarrollo social imperante. Ceuta. Pp. 27-29junio de 2008.

Delgado Noguera, M.A. (2009). ¿La educación física en crisis? Unas reflexiones personales. EmasF. Revista digital de Educación Física. http://emasf.webcindario.com/editorial_33.pdf

Delgado Noguera, M.A. (2009). Los estilos de enseñanza en la recreación. De la teoría a la práctica o de la práctica a la teoría. En Ruiz, F. Checa, J. J. y Ros, E., J (2009). Centro escolar promotor de actividad físico-deportiva-recreativa saludable. Respuestas a problemas de sedentarismo y obesidad. Ceuta. Pp. 207 - 224.

Delgado Noguera, M. A. (2010). La Educación Física y los Estilos de Enseñanzas Socializadores. Una forma de intervención en la educación en valores. II Congreso Nacional Fomento de actitudes éticas a través de la actividad física yel deporte. Deporte, Comunicación y ética. Facultad de ciencias de la actividad física y el deporte. Granada, 19-21 de noviembre 2010.

Delgado Noguera, M.A. (2014). Evolución de la Didáctica de la Educación Física: un repaso a los últimos cuarenta años. Vconvención de postgraduados del INEF de Madrid. Madrid, 28-29 de noviembre de 2014.

Devis Devis, J. (1996). Educación Física, deporte y currículum. Investigación y desarrollo curricular. Madrid: Visor.

Diem, L. (1969). ¿Quién es capaz de...?: Ejemplos para la educación del movimiento en los primeros grados de la escuela primaria. Madrid: Paidós.

Fernández Cruz, M. (1995). Ciclos en la vida profesional de los profesores Revista de Educación, 506, 153-203. Universidad de Granada.

Fernández Pérez, M. (1994). Las tareas de la profesión de enseñar. Práctica de la racionalidad curricular. Didáctica aplicable. Madrid: Siglo XXI.

Generelo, E. (1995). Seguimiento del compromiso fisiológico en una clase de deporte educativo en las primeras edades de educación primaria. (Tesis doctoral). Zaragoza: Universidad de Zaragoza.

Gil, J. (2009). Estrategias didácticas para mejorar la convivencia y participación del alumnado en Educación Física. (Tesis doctoral). Granada: Universidad de Granada.

González, C. et al. (2010). Los secretos del profesorado eficaz en Educación Física. Barcelona: INDE.

Guedea, J.C. (2009). Análisis de los estilos de enseñanza utilizados por los profesores de educación física del nivel primaria en la ciudad de 
Chihuahua. (Tesis doctoral). Granada: Universidad de Granada.

Huberman, H. (1989). Les phases de la carriere ensegnante. Revue Francaise de Pedagogie, 86.

Huberman, M. (1990). Las fases de la profesión docente. Ensayo de descripción y previsión. Qurriculum 2, 139-160.

Ley General de la cultura física y deportes. (1980). BOE núm. 89, de 12 de abril de 1980, páginas 7908 a 7913. http://www.boe.es/buscar/ doc.php?id=BOE-A-1980-7635

Ley 14/1970, de 4 de agosto, General deEducación y Financiamiento de la Reforma Educativa. (1970). BOE núm. 187, de 6 de agosto de 1970, páginas 12525 a 12546. http://www.boe.es/diario_boe/ txt.php?id=BOE-A-1970-852

Ley Orgánica 5/1980, de 19 de junio, por la que se regula el Estatuto de Centros Escolares. BOE núm. 154, de 27 de junio de 1980, páginas 14633 a 14636. http://www.boe.es/buscar/doc.php?id=BOE-A1980-13661

Ley Orgánica 8/1985, de 3 de julio, reguladora del Derecho a la Educación (1985). BOE núm. 159, de 4 de julio de 1985, páginas 21015 a 21022. http://www.boe.es/buscar/doc.php?id=BOE-A-198512978

Ley Orgánica 1/1990, de 3 de octubre de 1990, de Ordenación General del Sistema Educativo. BOE núm. 238, de 4 de octubre de 1990, páginas 28927 a 28942. http://www.boe.es/buscar/ doc.php?id=BOE-A-1990-24172

López Herrerías, J.A. (1978). Roles y funciones del profesor. Madrid: Edelvives.

López Jiménez, J.A. (2012). Calidad total de la materia de Educación Física: Adaptación del Modelo Europeo de Excelencia. El caso de los centros de Enseñanza Secundaria Obligatoria en la ciudad de Granaday su provincia. (Tesis doctoral). Granada: Universidad de Granada.

López Jiménez, J. A., Delgado Noguera, M. A. (2010). Cuestionario Profesorado. Calidad de la Educación Física. Granada: K\&L.

Manzano, I (director) Cañadas, F. Delgado Noguera, M. A., Gutiérrez, M., Sáenz - López, P., Sicilia, A. y Varela, R. (2003). Currículo, deporte y actividad física en el ámbito escolar. La visión del profesorado de Educación Física en Andalucía. Málaga: IAD.

Méndez, A. (1999). Análisis comparativo de las técnicas de enseñanza en la iniciación a dos deportes de invasión: el floorball patines yel Baloncesto. (Tesis doctoral). Granada: Universidad de Granada.

Montávez, M (2011). La expresión corporal en la realidad educativa. Descripción y análisis de su enseñanza como punto de referencia para la mejora de la calidad docente en los centros públicos de la educación primaria de la ciudad de Córdoba. (Tesis doctoral). Granada: Universidad de Córdoba.
Mosston, M. (1966). Teaching physical education. Columbus, OH: Merrill.

Mosston. M. (1978). Enseñanza de la Educación Física. Del comando al descubrimiento. Buenos Aires: Paidós.

Piéron, M. (1986). Pedagogía de la actividad física y deporte. Malaga: Unisport.

Rosales, C. (1992). Posibilidades de cambio en la enseñanza. (Perspectiva del profesor). Buenos Aires: Cincel.

Sánchez, F. Delgado Noguera, M.A. y otros (1976). Orientaciones para la programación de la Educación Físico-Deportiva en la EGB. Revista Didascalia. Revista de orientación didáctica e investigación pedagógica. 52, 5-49.

Sánchez Bañuelos, F. (1984). Bases para una Didáctica de la Educación Física y el Deporte. Madrid: Gymnos.

Santos Guerra, M.A. (1988). Patología general de la evaluación educativa. Infancia y Aprendizaje. Núm. 41.

Santos Guerra, M.A. (1993). La evaluación: un proceso de diálogo, comprensión y mejora. Málaga: Aljibe.

Sicilia,A.(1997). Evolución del contenido del pensamiento del estudiante de Educación Física, a través de un programa docente orientado hacia la autonomía de la enseñanza. Un estudio de casos en Bachiller. (Tesis doctoral). Granada: Universidad de Granada.

Sicilia, A. y Delgado Noguera (2002). Educación Física y Estilos de enseñanza. Barcelona: INDE.

Siedentop, D (1986). Developing Teaching skills in Physical Education. Palo Alto: Mayfield publishing Company.

Solana, A. (2003). Análisis y valoración de la responsabilidad de los alumnos de Bachillerato utilizandola microenseñanza para el aprendizaje de los deportes. (Tesis doctoral). Granada: Universidad de Granada.

Tonucci, F. (1984). Con ojos de niño. Barcelona: Barcanova.

Vernetta, M.(1994). Efecto diferencial de tres estrategias en la práctica para el aprendizaje de habilidades gimnásticas. (Tesis doctoral). Granada: Universidad de Granada.

Yoyce, B. Weil, M, y Calhoun, E. (2002). Modelos de Enseñanza. Barcelona: Gedisa.

Zabalza Beralza, M.A., Zabalza Cerdeiriña, M.A (2011). Profesores y profesión docente. Madrid: Narcea Ediciones.

\section{Webgrafía}

Spectrum Institute for Teaching and Learning (2012). Spectrum of Teaching Styles http://www.spectrumofteachingstyles.org/ Recuperado noviembre 2014.

Rossaro,A.L., (2008). Educación 0. http://www.educdoscero.com/2011/ 10/una-pisca-de-actitud-20-para-ser.html Recuperado enero 2015
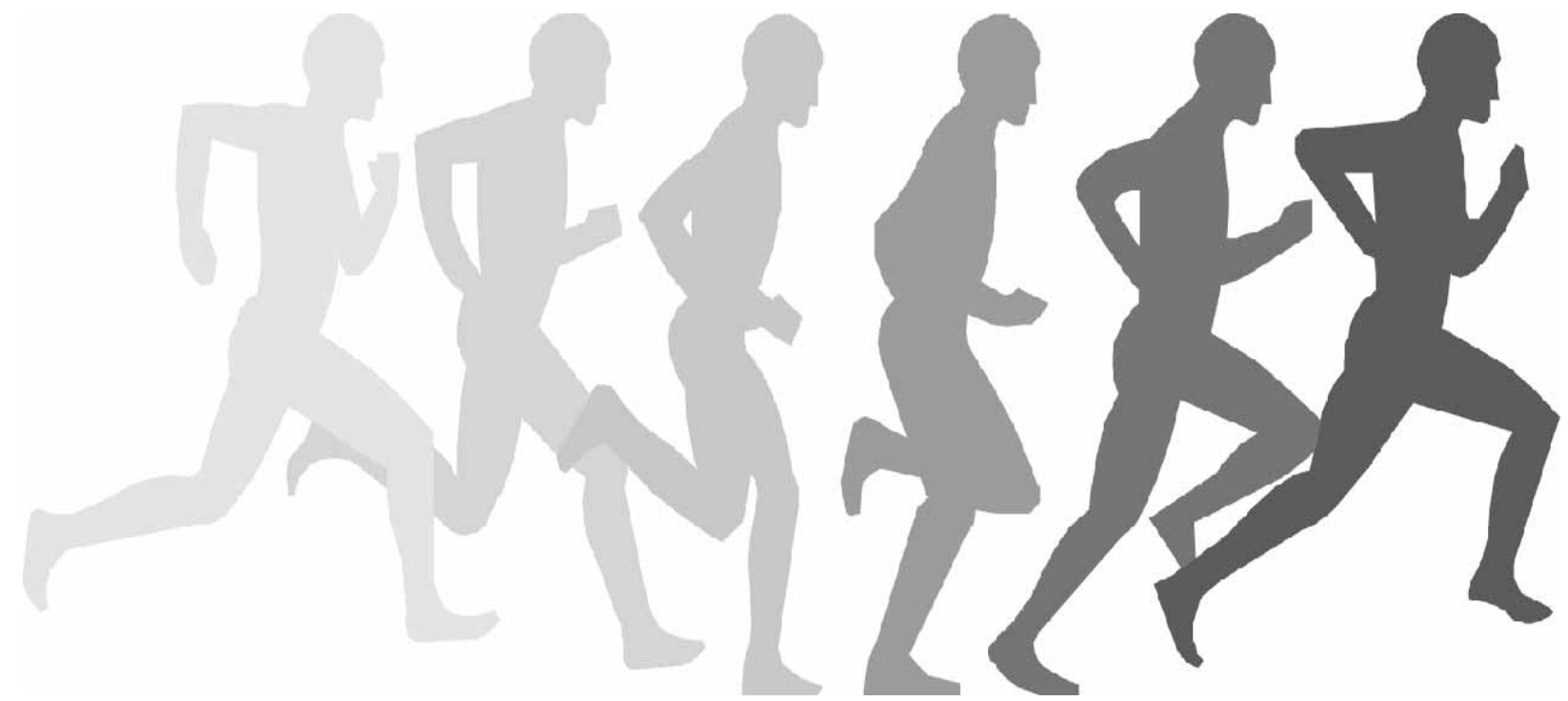